\title{
Risk of death after release from prison: a duty to warn
}

\author{
Deborah Harding-Pink, Oldrich Fryc
}

University Institute of Legal Medicine, Geneva Deborah Harding-Pink, MB, research registrar

Oldrich Fryc, MD, privat-docent

Correspondence to: Dr D Harding-Pink, Institut Universitaire de Médecine Légale, 1211 Geneva 4, Switzerland.
Prisoners have been considered to be a vulnerable group, having high levels of morbidity on entry to prison' and raised indices of social and psychological dysfunction. ${ }^{2}$ Mortality from suicide is higher among prisoners than in the general population. ${ }^{\text {T This report }}$ concerns deaths of people after leaving prison.

\section{Methods and results}

Geneva prison (for men and women) has 300 beds and serves as a remand prison and for short and medium term sentences. One fifth of the prisoners are dependent on opiates at entry. For our study we cross checked prison medical records with medicolegal necropsy reports in the Canton of Geneva.

During 1982-6, 102 sudden deaths among former prisoners were recorded, of which a large number occurred during the first year after release as compared with subsequent years (table). Mortality from sudden

Deaths from poisoning or from other causes stratified by length of surcizal after release from prison

No of vears since release from prison

$123+5 \quad 6 \quad 7 \quad 8 \quad 9 \quad 10 \quad 11 \quad 12 \quad 13 \quad 1+\quad 15 \quad 16 \quad 17$

Deaths from poisoning Deaths from other causes $\begin{array}{lllllllllllllllll}26 & 3 & 5 & 5 & 2 & 0 & 0 & 0 & 0 & 0 & 0 & 0 & 0 & 1 & 0 & 0 & 0 \\ 13 & 6 & 6 & 5 & 7 & 5 & 2 & 2 & 3 & 2 & 0 & 1 & + & 3 & 0 & 0 & 1\end{array}$

deaths during the first year after release was estimated as 4.8 deaths $/ 1000$ person years, a rate over four times the age adjusted rate in the general population. Deaths by poisoning accounted for 26 of the 39 deaths $(66 \cdot 7 \%)$ during the first year compared with 16 of 63 deaths in subsequent years $\left(\chi^{2}=16.9 ; \mathrm{df}=1 ; \mathrm{p}<0 \cdot 01\right)$. Compared with a sample of consecutive prison entries ex-prisoners dying within one year of release had a higher average number of prison sentences $(4.7 v 1.7)$ and a higher incidence of drug dependence $(62 \% v$ $20 \%$ ).

The mortality among drug users during the first year after release was estimated as 15 deaths/1000 person

years. Thirteen of the 26 deaths by poisoning occurred during the first 45 days after release. This clustering represents a mortality for the period equivalent to about 60 deaths/ 1000 person years. All deaths in this initial period were due to poisoning by heroin or morphine or methadone or both: alcohol and benzodiazepines were also often found at necropsy. Methadone was considered to be the main cause of six deaths, all occurring within 14 days of release from prison.

\section{Comment}

Our mortality data were limited geographically and by cause of death, so that deaths of ex-prisoners outside the canton or due to natural causes were not recorded. Had these data been available the observed mortality would have been higher. Despite this limitation, the striking feature was the high number of deaths from opiate poisoning during the first year after release from prison and especially during the early weeks after release. Loss of tolerance may have been a risk factor ${ }^{+}$ together with coincidental misuse of other drugs. The role of methadone seemed to be especially important. Whether this was due to ignorance about the quantity of drug to use, the concomitant effect of other drugs, or whether methadone was more easily available than heroin is not known.

Release from prison must be considered a stressful event, especially for drug abusers who leave with decreased or absent tolerance but still with social and psychological problems, so that further drug use is highly likely. 'There therefore seems to be a clear duty for prison medical staff to warn all drug abusers about to leave prison of the risk of overdose if they take doses which normally they could tolerate. This means teaching addicts to estimate the dose appropriate to their state of tolerance and about the risks entailed by using unfamiliar drugs and by multiple drug abuse.

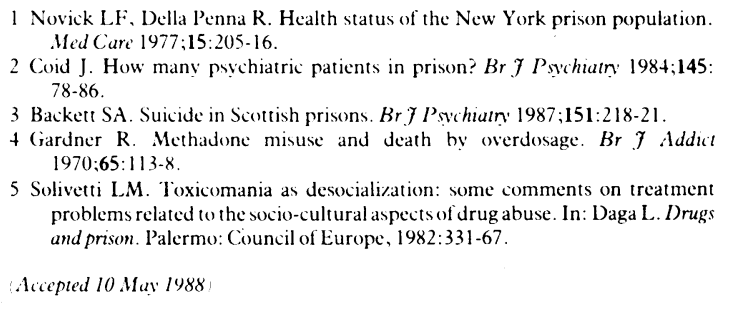

\section{The frozen hip: an underdiagnosed condition}

\author{
M D Chard, J R Jenner
}

\section{Department of \\ Rheumatology, \\ Addenbrooke's Hospital, Cambridge CB2 2QQ \\ M D Chard, MRCP, senior registrar \\ $\mathrm{J}$ R Jenner, MD, consultant rheumatologist}

Correspondence to: Dr Chard.
Capsulitis or frozen shoulder is a well recognised

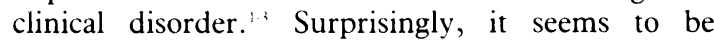
exclusive to the shoulder joint. The cases described suggest that an analogous disorder may also affect the hip and is probably underdiagnosed.

\section{Case reports}

Case 1-A 55 year old man presented with a two month history of pain of spontaneous onset in the right hip. Examination showed a painful restriction of active and passive hip movements with rotation being virtually absent. Full blood count, erythrocyte sedimentation rate, serum uric acid concentration, the results of liver function tests, serum calcium concentration, and Rose Waaler and antinuclear factor were all normal, as was a plain radiograph of the hip.
An isotope bone scan (technetium " diphosphonate) showed increased uptake around the right hip. The results of hip aspiration and arthrography were normal. Six months after onset the pain started to ease, and this was followed by a gradual return of movement over the next year.

Case 2-A 41 year old man with maturity onset diabetes presented with a six week history of pain of spontaneous onset in the right hip, worse at night and aggravated by bearing weight. His movements were not initially restricted, but one week later flexion was limited, with a pronounced restriction of rotation. The results of all blood tests, as in case 1, were normal. Plain radiograph and aspiration of the hip yielded normal results. An isotope bone scan showed a high uptake in the region of the femoral head and the trochanteric area. After three months pain at night had settled with some improvement in the range of movement. After eight months symptoms had settled and examination yielded normal results.

Case 3-A 41 year old woman presented with a four month history of gradually increasing hip pain. Examination showed only mild restriction of flexion and extension but pronounced limitation of rotation of the hip. Laboratory investigations and plain 
radiographs of the hips yielded normal results. Computerised tomography of the pelvis and hips, a hip aspiration, and an arthrogram also yielded normal results. For three months there was no clinical improvement but one year after onset the pain had settled and full function had returned despite mild restriction of range.

All three patients were followed up for over one year after resolution and none developed any other joint disorder.

\section{Comment}

The three patients described were middle aged and developed hip pain of spontaneous onset and joint stiffness mainly affecting rotation. No evidence of a systemic disorder, local infection, or a lumbar spine problem was found. The isotope bone scans performed on two patients showed an increased uptake despite a normal erythrocyte sedimentation rate consistent with capsulitis. Spontaneous resolution of pain after some months was later followed by the return of movement. One patient was diabetic. The similarities to the clinical frozen shoulder are striking.

In the only previous report of a stiff hip condition believed to be due to capsulitis two patients were reported on who had frank capsular retraction on arthrography. ${ }^{+}$In the two patients in this report who had arthrography this was not found. Capsular retraction on arthrography is often not found in patients with frozen shoulders and is not essential for diagnosis, which is made on clinical grounds.

We suggest that the "frozen hip" is a clinical entity, which can be distinguished from the "irritable hip" syndrome, which causes pain but only slight restriction of movement. Clinically, a frozen hip shows limitation of active and passive movements, rotation being particularly affected. Investigations to exclude systemic disease must yield negative results, but an isotope bone scan will show an increased uptake. Once the condition is diagnosed spontaneous resolution can be expected and the patient given a favourable prognosis. A detailed prospective study of such a group of patients should improve understanding of the phenomenon of joint capsulitis.

1 Reeves B. The natural history of the fro\%en shoulder syndrome. Scand 7 Rheum 1975:4:193-6.

2 Bulgen DY, Binder AI, Hazleman BL. Frozen shoulder: prospective clinical study with an evaluation of three treatment regimens. Ann Rheum Dis 1984;43:353-60.

3 Binder AI, Bulgen DY, Hazleman BL, Roberts S. Frozen shoulder: a long-term prospective studv. Ann Rheum Dis 1984:43:361-4.

Caroit M, Diian A, Hubault A. Normandin C. Deux cas de capsulite retractile de la hanche Rer Rheum Mal Osteour 1963:30:784-9.

Binder AI, Bulgen DY, Hazleman BL, Tudor J. Wraight P. Frozen shoulder: an arthrographic and radionuclear scan assessment. Ann Rheum Dis 1984;43: $365-9$.

Accepted 21 April 1988

\title{
Does wearing two pairs of gloves protect operating theatre staff from skin contamination?
}

\author{
H Matta, A M Thompson, J B Rainey
}

Department of Clinical Surgery, University of Edinburgh, Royal Infirmary of Edinburgh, Edinburgh EH3 9YW

H Matta, MB, registrar A M Thompson, $\mathrm{MB}$, honorary registrar

J B Rainey, CHM, lecturer in clinical surgery

Correspondence to: $\mathrm{Dr}$ Matta.
The effectiveness of surgical gloves in preventing contact between the surgeon's skin and the patient's tissue or body fluids during surgery has been questioned recently. ${ }^{\prime 2}$ Although needlestick injuries that penetrate both glove and skin are painfully obvious, 'it is more common for gloves to be punctured without the wearer's knowledge, which may result in prolonged contamination of the skin with potentially infective material. ${ }^{2}$ Double gloving (wearing two pairs of gloves) is generally adopted by surgical teams operating on high risk cases and aims at reducing the risk of such incidents. We tested the effectiveness of this precaution in maintaining an intact barrier between the patient and the surgical staff.

\section{Methods and results}

Ten surgeons and nine scrub nurses in a surgical unit wore two pairs of gloves during general surgical operations on 144 consecutive patients. The gloves were tested at the end of the operation by a recognised method of detecting perforation. ${ }^{3+}$ Each glove was filled with one litre of water and subjected to external

Numbers of punctures detected at different sites in 728 outer and inner gloves

\begin{tabular}{lccccc}
\hline & \multicolumn{2}{c}{ Left hand } & & \multicolumn{2}{c}{ Right hand } \\
\cline { 2 - 3 } \cline { 5 - 6 } \cline { 5 - 6 } & Outer glove & Inner glove & & Outer glove & Inner glove \\
\hline Thumb & 6 & & & 1 & \\
Index finger & 33 & 5 & & 4 & 1 \\
Third finger & 6 & 1 & & 2 & 1 \\
Fourth finger & 3 & & & 5 & 1 \\
Fifth finger & 1 & & & & \\
Palm & 16 & 6 & &
\end{tabular}

compression with the open end occluded. Punctures were indicated by a fine jet of water and their number and site noted for both the inner and outer gloves. The subject's role (surgeon, assistant, or scrub nurse) and grade were also recorded together with the duration and nature of the operation. Before the gloves were tested the subjects were asked if they were aware of any damage to the gloves. Data were analysed with the $\chi^{2}$ test.

Punctures were detected in $77(11 \%)$ of the 728 outer gloves tested (table) and occurred more frequently in those worn by surgeons $(52 / 288,18 \%)$ than in those worn by assistants $(12 / 254,5 \% ; \mathrm{p}<0.001)$ or nurses $(13 / 186,7 \% ; p<0.005)$. Fifteen of the 77 inner gloves worn in these cases were also punctured, giving an overall rate of puncture of inner gloves of $2 \%$; again the rate was higher for surgeons (13 inner gloves were punctured: 52 outer gloves, $25 \%$ ) than for assistants $(1: 12,8 \%)$ or nurses $(1: 13,8 \%)$. No punctures were found in a control group of 20 unused gloves chosen at random or in inner gloves worn with outer gloves that were found to be intact when tested.

Most punctures (65) occurred in the left glove, particularly the index finger (33). Before testing 38 of the punctures had not been detected by the subjects.

The grade of the subject wearing the gloves, the duration of the operation, and whether the operation was elective or an emergency procedure did not have any effect on the puncture rate.

In 37 operations the outer gloves were removed before the end because of discomfort or loss of sensitivity, surgeons being more intolerant (all 37 of these operations $(26 \%))$ than assistants $(20(14 \%) ; \mathrm{p}<0.02)$ or nurses $(11(8 \%) ; \mathrm{p}<0.01)$.

\section{Comment}

This study confirms that wearing two pairs of gloves confers some protection against contamination of the skin with patients' tissue and fluids. The rate of puncture of outer gloves of $11 \%$ is similar to that reported previously ${ }^{3+}$ but a third of that in a recent study. ${ }^{2}$ Surgeons seemed more at risk than assistants or nurses. The rate of perforation of inner gloves was only 